Int. J. Dev. Biol. 50: 315-322 (2006)

doi: $10.1387 / \mathrm{ijdb} .052062 \mathrm{vs}$

\title{
The natural variability of morphogenesis: a tool for exploring the mechanics of gastrulation movements in amphibian embryos
}

\author{
VICTORIA A. SCOBEYEVA* \\ Department of Biological Evolution, Faculty of Biology, Moscow State University, Moscow, Russia
}

\begin{abstract}
The investigation of natural variability of metric morphological characters in frog gastrulation revealed that in genetically and environmentally homogeneous samples of embryos their variability is of a higher order of magnitude than that known for quantitative metric characters in adult organisms. Matching the coefficients of variation of characters under consideration to the specific rates of their changes in normal development revealed a strong positive correlation between the rates of morphological change and the amount of morphological variance. The increase in the variance is mainly in characters concerned with shaping of moving embryonic areas and arises as a result of a positive feedback between the movement of a given area and recruitment of cells from surrounding areas into the movement. The account of natural variation suggests a new model of amphibian gastrulation whose essential feature is the intimate connection between the movement and shaping of the dorsal blastopore lip of the gastrula.
\end{abstract}

KEY WORDS: morphogenesis, variability, gastrulation, amphibian

Introduction: the morphogenetic component of natural variability

The idea that the statistic analysis of metric morphological characters in embryonic structures in status nascendican help in understanding the nature of embryonic morphogenetic fields was once stated by A. G. Gurwitsch $(1911 ; 1922)$ and, independently, by R. Gordon (1999). Our investigations (Cherdantsev, Scobeyeva, 1994; 1996; Cherdantseva, Cherdantsev, 1994; Scobeyeva, Cherdantsev, 1999) confirmed that morphological variation of embryonic structures at the onset of their formation is of a higher order of magnitude than that in their differentiated state. The normal morphogenetic course proves to be subject to fluctuations whose scale is comparable to that of regulative changes arising in the developing system as a result of experimental intervention into normal development. Thus the normal individual variability of morphogenesis can be treated as a "natural experiment" with the obvious advantage over usual lab experiments being precisely the absence of external intervention. When a developing system is at a threshold of initiating new developmental pathways (Waddington's chreods, see Waddington, 1940), the current morphogenetic interactions are replaced by new ones. At such a time response to experimental influences is strongly non-linear, which means that there is a loosening of the connection between cause and effect in the behavior of the developing system (Spemann, 1936; Waddington, 1940). On the other hand, new trends in variation become apparent (Cherdantsev et al., 1996). It follows that the statistic analysis of the scales and directions of variability can yield information that is missed by the experimental approach.

Insofar as normal morphogenesis is subject to individual variation even in genetically homogeneous groups of embryos developing in fairly equivalent and optimal environmental conditions, this variation can be unambiguously attributed neither to the genetic, nor to environmental causes (see Martin, 2005; Fraga et al., 2005). The morphological variation of development merits being isolated as a special component of general phenotypic variance - a morphogenetic component - which means that the variability is inherent to the directional morphogenetic changes occurring in the developing system both in space and time (Cherdantsev, Scobeyeva, 1994).

To bridge the gap between the morphogenetic component and variation in definitive morphological characters, recall that what in

Abbreviations used in this paper: DBL, dorsal blastopore lip; ES, epithelial sheet; MZ, marginal zone; YP, yolk plug. 
quantitative genetics is called the environmental variance embraces, by definition, all variation of non-genetic origin. In adults the closest concept to the morphogenetic component is repeatability, when more than one measurement of a morphological character can be made in each individual in space and/or time and the phenotypic variance can be partitioned into variance within individuals and variance between individuals (Falconer, 1981). The same partitioning of variance is basic to the analysis of fluctuating asymmetry, a concept that can be considered as a subset of repeatability (Sullivan et al., 1993). Both components of variance are usually considered as entirely environmental in origin, one (between-individual) being attributed to permanent and non-localized circumstances and the other (within-individual) to temporary and localized developmental variation.

There are two assumptions implicit in the idea of repeatability (see Falconer, 1981). One is that the variances of different measurements of the same character made in the repetitive parts of the organism are equal and have their between- and withinindividual components in the same proportion. In reality, however, this is may not be the case because spatially repeated characters are subject to correlation between their mean values and their coefficients of variation. Thus, the amount of variance in the number of bristles on the ventral surfaces of the abdominal segments in Drosophila melanogasterdepends both on the mean number of bristles and their spacing in a given a segment (Reeve,
A
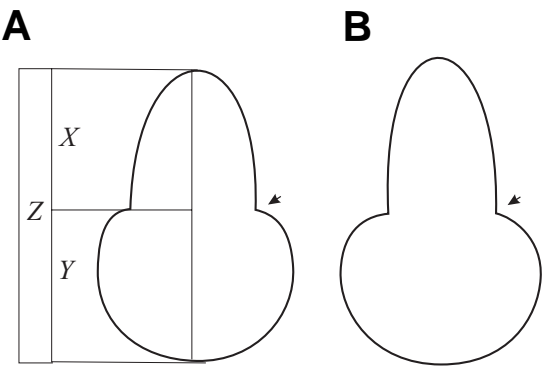

E

D

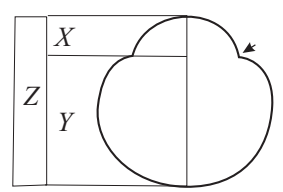

G

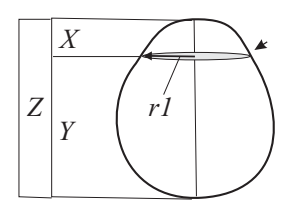

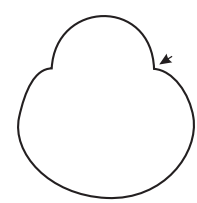

H

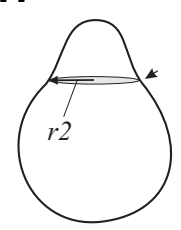

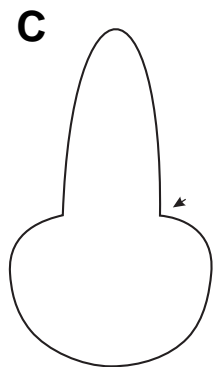

F

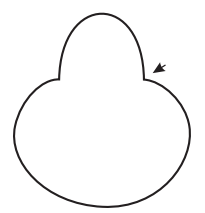

I

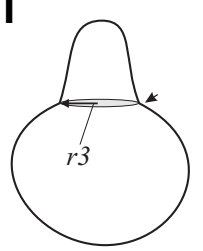

Fig. 1. Scheme illustrating the variation patterns in the imaginary example of morphogenesis. (A-C) Variation series of differentiated forms. (D-F) That of embryonic forms with no morphogenetic component. (G-I) That of embryonic forms with morphogenetic component. $X, Y, Z$, measurements characterizing the shape; arrows, outgrowth separation boundary positioning; r1-3, radii of curvature; for other explanations, see text.
Robertson, 1954).

The other assumption is that a character in the line up of repeatable, but differently molded structures, is the "same" phenotypic character, at least from the genetic point of view (Falconer, 1981). Meanwhile, at the heart of morphogenetic component isolation lies the distinction between characters and structures (Cherdantsev etal., 1996). "Structuring" means that characters which in the initial state could be actualized everywhere in the system, become restricted to only a part of the system. This is the only way in which a part of the system - but not the characters of the part- becomes a new structural domain, which means that morphogenetic interactions emerge on a new macroscopic scale (Cherdantsev et al., 1996). The variation arising as a result of actualizing the repetitive characters everywhere in the system is replaced by their joint variation inside the domain on a size scale of the domain and time scale of its shaping. Thus, the origin of a new domain implies the change not in morphological characters, but rather in the spatiotemporal scale of their variation. If and only if, this is the only reason for the change in the variation pattern, then the origination of new variation canals can be attributed to the morphogenetic component of variation. In terms of the quantitative genetics this means that the between-individual variance increases at the expense of within-individual one.

To illustrate this point, consider a simple imaginary example (Fig. 1). Consider a spherical shell, whose robust shape is equivalent to that of an ellipsoid with nearly equal long and short axes. Let the shell be subject to shaping so as to form an outgrowth whose shape differs from that of the initial sphere both in the absolute curvature and proportion of the long and short axes. Assume that in the differentiated state, with a series of variations as shown in Figs. $1 \mathrm{~A}-\mathrm{C}$, there is no statistic correlation between $Y$, the outgrowth length and $X$, the length of the remaining spherical shell. Then, $Z$, the length of the sphere with the outgrowth, is the sum of $X$ and $Y$, so that the variance of $Z, \sigma 2 Z$, is partitioned into the variances of $X$ and $Y, \sigma 2 X$ and $\sigma 2 Y$, where $\sigma 2 Z=\sigma 2 X+\sigma 2 Y$. This means that the variation of $Z$ is simply a sum of the variation of its independently varying components, whose relative contribution to the general variance depends on the coefficients of correlation between $Z$ and $X$ and $Z$ and $Y$ (Wright, 1923).

Figs. 1D-F show a variation series reflecting the same relationship between $Z$ and its components, $X$ and $Y$, but referring to the outgrowth formation. The identity of relationship between the parts of developing and differentiated systems means, as shown in Figs. 1D-F, that the outgrowth area is separated off before the onset of its shaping and so there is no correlation between shaping of this area and how its separation boundary moves, shown in this series of figures by arrows. With this formation mode the outgrowth area is not a domain in the sense outlined above, as its origination does not introduce any new scale of withinindividual variation, as compared to the differentiated state. The model shown in Figs. 1D-F shares the basic premises of the positional information paradigm that establishing and interpreting spatial coordinates are independent processes (Wolpert, 1969). It is worth mentioning in this context is that the quantitative analysis of variance of the expression of genes influencing the segment patterns in the embryogenesis of Drosophiladoes not fit in well with this paradigm (Jaeger et al., 2004).

The variation series of embryonic forms, shown in Figs. 1G-I, 
A
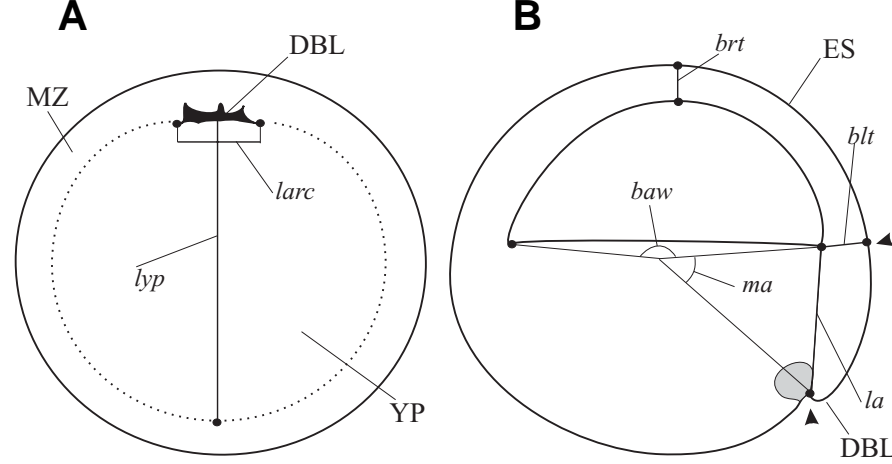

C

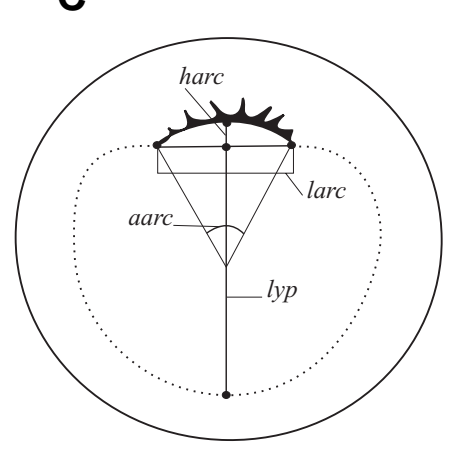

D

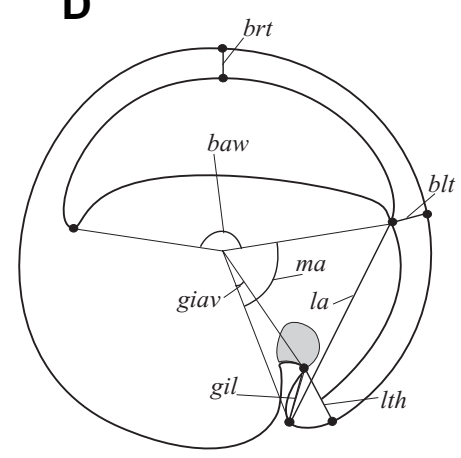

Fig. 2. Scheme of measuring the metric morphological characters which characterize the dynamics of frog gastrulation. $(\mathbf{A}, \mathbf{B})$ The initiation ofgastrulation. (C,D) Formation of the dorsal blastopore lip. $(A, C)$, vegetal views; $(B, D)$ anatomical sagittal sections; dotted lines, the separation boundary between the yolk plug and marginal zone; shading, the archenteron bottom; arrowheads in (B) show the marginal zone boundaries; for designation of characters, see text.

originates with an alternative model of outgrowth formation based on a presumption that establishing the domain-separation boundary and shaping of the domain are not independent processes. Each step of outgrowth shaping molds a new separation boundary between the outgrowth and the rest of the shell. This means that the between-individual variation in positioning of the domain separation boundary arises as a result of within-individual differences between the outgrowth shape and that of the shell as a whole. In other words, one can read this variation series as representing the succession over time of moving the domain separation boundary within the same individual. Then, measuring the radii of curvature of the outgrowth separation boundaries $R 1$ $R 3$ in different individuals, shown in Figs. $1 \mathrm{G}-\mathrm{I}$, yields the same between-individual variance as obtained from measuring the within-individual variance of the shell curvature inside and outside the outgrowth domain. It follows that we must add to the initial components of $Z$ variance $\sigma 2 x$ and $\sigma 2 Y$, the covariance components $\operatorname{Cov}(X, Y)$ and $\operatorname{Cov}(Y, X)$, so that $\sigma 2 Z=\sigma 2 X+\sigma 2 Y+\operatorname{Cov}$ $(X, Y)+\operatorname{Cov}(Y, X)$ where $\operatorname{Cov}(X, Y)$ and $\operatorname{Cov}(Y, X)$ have positive values. With this addition, as compared to what is shown in Figs. $1 \mathrm{D}-\mathrm{F}$, variation arises as a result of the positive correlation between shaping the domain and recruitment into this shaping of the adjacent shell areas. It is this additional variance that is characteristic of the morphogenetic component.

The aim of this work is to analyze the morphogenetic component in the amphibian gastrulation using the natural variation in metric characters as a tool for investigating the dynamics and spatial pattern of gastrulation movements. To be convinced that we are dealing with the normal variability of morphogenesis and not with environmental or genetic deterioration of development, it is better to work with egg clutches spawned in unpolluted natural ponds and developed in conditions which permit us to exclude density-dependent factors of embryonic variation and mortality. The egg clutches of a common frog, Rana temporaria L., provide an opportunity to consider all morphological variations, irregardless of scale, as reflecting the normal course of gastrulation, so long as we achieve optimal conditions in which embryonic mortality does not exceed 1-2\% (Cherdantsev, 2003).

The special attention in this work will be paid to the fact that the development in the embryo of a new structural domain, the dorsal blastopore lip of gastrula, generates new variation trends. Analyses based on the routine methods of multivariate statistics permits us to reconstruct both the geometry and dynamics of mass cell movements.

\section{The quantitative dynamics of metric characters and the movement-shaping model of amphibian gastrulation}

Although the cell movements in amphibian gastrulation seem to have been studied in minute detail (see, for review, Beetschen, 2001), this cannot be said about the geometry of cell movement on a macroscopic scale. The choice of metric characters that change in the course of gastrulation is based, first, on their accessibility for accurate measurement and, second, on the possibility of matching their changes to that in the shape of embryonic regions.

The selection of measurements made in the frog gastrula at the onset of gastrulation is shown in Figs. $2 A, B$ : $A$ is the vegetal side view, $B$ is the anatomical section of embryos fixed in $8 \%$ formaldehyde. The macroscopic subdivision of the embryo at the onset of gastrulation is into three parts, with separation boundaries shown by arrowheads in Fig. 2B (see also Fig. 2A): the epithelial sheet (ES) circumscribing the blastocoel, the marginal zone (MZ) and the yolk plug (YP) consisting of yolky endoderm cells. These anatomical parts correspond not to the fate map areas, but rather to structural domains with a distinct developmental history (Keller, 1996; Cherdantsev, 2003). Thus the ES originates from the animal hemisphere of the early blastula, the $\mathrm{MZ}$ develops from the descendants of vegetal blastomeres bordering the circumference of the animal hemisphere, while the YP corresponds to the rest of the vegetal hemisphere.

The characteristic feature of the frog embryo at the initiation of gastrulation movements is that the archenteron anlage arises as a furrow whose curvature, as shown in Fig. $2 \mathrm{~A}$, is very close to that of the arc of the latitudinal $M Z$ circumference along which this furrow appears. It follows (cf. Hardin and Keller, 1988; for details see Cherdantsev, 2003) that in the initiation phase the arc of the dorsal blastopore lip (DBL), shown in Fig. 2A, has no curvature on its own. Therefore, in order to estimate its shape, it is sufficient to measure the linear (larc) and angular (aarc) arc lengths. The relationship between the $M Z$ and $Y P$ (the degree of epiboly) is characterized by the longitudinal YP length (Iyp).

In the anatomical section of the embryo in the initiation phase 
A

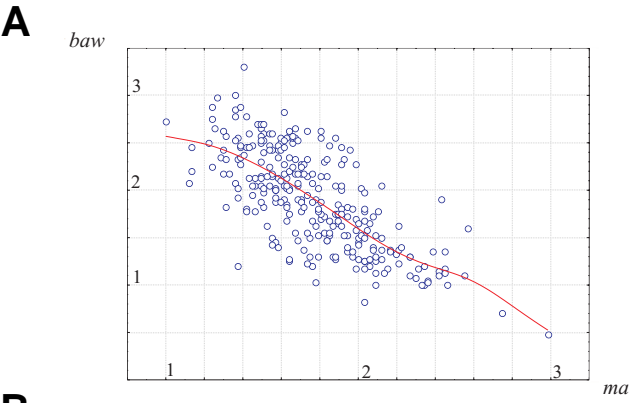

$B_{\text {lare }}$

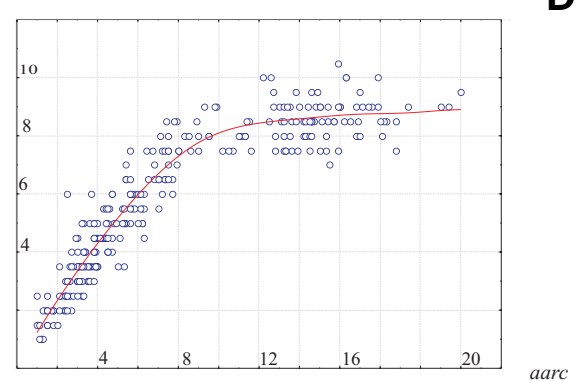

$\mathbf{C}_{h a c}$

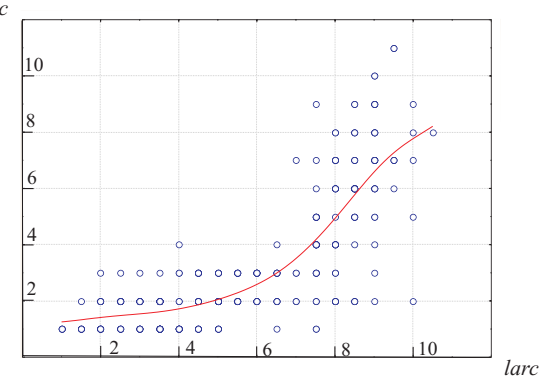

gil

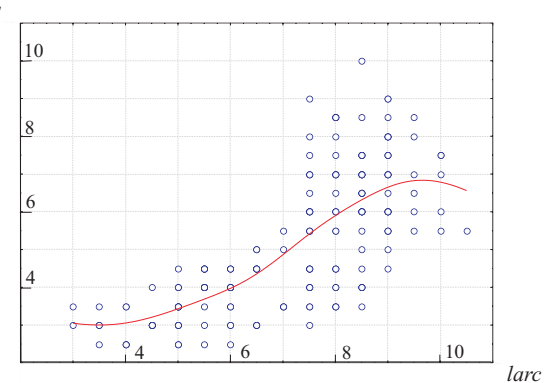

Fig. 3. Interaction of morphological variables characterizing gastrulation morphodynamics. (A-

D) The distribution of measurements; shown in red are the curves calculated by the method of least squares; for each variable its values are those normalized to the lowest one. (A) Blastocoel angular width (baw) as dependendent on MZ angulal length. (B) Linear DBL arc length (larc) as dependent on its angular length (aarc). (C) DBL arc height (harc) as dependent on DBL arc linear length (larc). (D) Linear archenteron length (gil) as dependent on DBL arc linear length (larc).

(see Fig. 1B) the shape of the animal ES is characterized by the blastocoel angular width (baw), blastocoel roof thickness ( brt $)$ and blastocoel lateral wall thickness $(b / t)$ at the dorsal separation boundary between the $\mathrm{ES}$ and $\mathrm{MZ}$, while the longitudinal length of the $\mathrm{MZ}$ is characterized by the angular $(m a)$ and linear $(m)$ distances between the archenteron anlage furrow and the boundary between the ES and MZ (see Fig. 1B). As for the DBL, in the initiation phase it is not sufficiently shaped to be measured. The archenteron furrow bottom (shaded in Fig. 1B) consists of bottle cells that initiate archenteron formation.

The next phase of gastrulation in which the DBL becomes a new morphological and morphogenetic gastrula domain is shown in Figs. 2C, D. In the vegetal side view (see Fig. 2C) the DBL arc cannot be inscribed into a single latitudinal circumference of the whole gastrula, which means the arc has acquired its own curvature. Therefore, the linear and angular arc lengths (larcand aarc) have to be supplemented by the arc height (harc). In sagittal section (see Fig. 2D) the DBL contour forms what in the theory of smooth mappings is called a fold (Mather, 1955). In common geometric terms this means that the convex surface of the dorsal archenteron roof is conjoined with the concave surface of the archenteron bottom. The latter, shaded in Fig. 2D, corresponds to the archenteron anlagen furrow, shaded in Fig. 2B. All the other measurements refer not to the DBL domain, but to the whole gastrula and are the same as in the initiation phase.

Quantitative analysis was made on a sample that included all embryos from successive fixations made from the same clutch of Rana temporaria eggs. The embryos were fixed at equal time intervals $\left(3 \mathrm{~h}\right.$ at $\left.16^{\circ} \mathrm{C}\right)$. The first fixation was made at the onset of gastrulation, at the stage shown in Figs. 2A, B; the second fixation corresponded to a stage at which the DBL acquired a crescent- topore closure.

This generally accepted model fits in well to the quantitative dynamics of metric characters that refer to the whole gastrula, except those referring to the DBL itself. Fig. $3 \mathrm{~A}$ shows the connection between the blastocoel angular width (baw, ordinate) and the $\mathrm{MZ}$ angular length (ma, abscissa). As shown in Fig. 3A, baw depends on ma in an even way. Here the even dependence means that the coefficients of correlation between the dependent and independent variable do not differ significantly in different abscissa intervals. This suggests that the epiboly mechanism is uniform throughout gastrulation. The course of epiboly seems to ignore the origin of the new morphogenetic domain (DBL). The other mutual dependencies of other epiboly components ( $m$, lyp, blt and brt, data not shown) are of the same type.

It follows, in the full agreement with the observations of Hardin and Keller (1988, see also Keller, 1996), that the epiboly movement in amphibian embryos is not connected with shaping of either MZ/YP separation boundary, or the outer embryonic surface which this boundary is moving over. This means, as shown in Fig. 4A representing the vegetal view of the whole gastrula, that at each step of epiboly the movement of cells or, rather to say, the direction of cell flows on the embryonic surface, is along the normal radii of curvature of the $\mathrm{MZ}$ circumference, whose shape remains similar to that of a circle. This is not to say that epiboly is a radially symmetric movement. In fact, its dorsoventral asymmetry allows for the formation of both the anteroposterior (AP) and dorsoventral (DV) embryonic axes. If we assume that this asymmetry, as proposed for the first time by Keller (1987; 1996), is based on the planar intercalation of cells, shown in Fig. 4A by dotted arrows, this does not necessarily affect the steady circular shape of the MZ-YP separation boundary. Both in time and space, 
the scale of each intercalation step is small enough, as compared to that of separation-boundary movement, so that there is no connection between the movement and shaping of this boundary. Then and only then, can one state that, on the scale of the whole gastrula, the cell movement tracks of epiboly, in spite of intercalation, correspond to the normal radii of curvature of the $M Z$ circumference. It will be obvious to see (for details: Cherdantsev, 2003) that only on this assumption one can consider the movement and shaping of embryonic areas on the whole gastrula scale as independent processes, as in the model, shown in Fig. 4A. As for the characters not concerned with the DBL movement, but rather with that of epiboly, this assumption seems to be valid.

This, however, cannot be extended into DBL behavior. Fig. 3B shows the dependence between the linear (larc, the ordinate) and angular (aarc, the abscissa) lengths of the DBL arc. This dependence is not even, as both the slope of the empirical curve obtained by the method of least squares and coefficients of correlation between the ordinate and abscissa decrease with the progress of DBL. To explain this, one has to assume that the arc's curvature is subject to an increase which is additional to that predicted by the model, shown in Fig. 4A, in which the arc moves as a part of the $\mathrm{MZ}$ circumference.

Fig. $3 \mathrm{C}$ shows how the DBL arc linear length (larc, the abscissa) is connected with the arc linear height (harc, the ordinate). The empirical curve, shown in this figure, represents almost a threshold dependence of the arc height on the arc length which means that that the arc forms a curvature on its own without respect to the course of epiboly.

It follows that the DBL movement is not simply a change both in the arc size and position, as could have been expected from the model shown in Fig. 4A. Rather, it could be portrayed as a movement-shaping based on feedback between the change in the arc position and shaping of the arc. The central assumption of the model shown in Fig. $4 \mathrm{~B}$, in the same vegetal view of the gastrula as Fig. 4A, is that the cells move not in the constant directions corresponding to that of the vector field vectors imposed beforehand on the mass cell movement, but instead, the cell flows, whose directions are shown in Fig. 4B by the arrows, adjust their slopes to the DBL contour which they mold and vice versa. Obviously this is not to say that the cell movement tracks are not matters of change in the model, shown in Fig. 4A, as this model assumes that there is a planar intercalations of cells in a direction that is perpendicular to the mass cell movement axes. The basic difference is in that the intercalation implies the returning of mass cells flows to their initial directions on the whole gastrula scale immediately after the occasional, though inevitable, distortion. This is the reason why, in spite of intercalation, the mass cell flows are directed along the normal radii of curvature of $\mathrm{MZ}$ circumference, with no respect to $\mathrm{DBL}$ formation. In contrast to that, the movement-shaping model, given in Fig. 4B, is based on the assumption that the cell flows regulate their directions by changing the DBL shape.

As shown in Fig. 4B, the movement-shaping model assumes that the cell flows deviate from the normal radii of curvature of the DBL arc. Consequently, as the cells enter the arc, they change the arc shape, while the arc shaping establishes a new orientation of cell flows, adjusting them to the new DBL shape. It is easy to see that the shaping component of DBL movement allows for the additional increase in the arc height, as compared to that following from the epiboly movement in which the DBL would have been remained simply a fragment of the $M Z$ circumference. The proportion of this increase is a positive function of deviation of cell flows from normal orientation. As follows from the data shown in Figs. $3 B, C$, the deviation is attenuated at later stages of DBL development, which means the shaping component of DBL movement degenerates with the completion of DBL formation.

Now let us return to the model, shown in Fig. 4A, whose basic premises is that the movement and shaping of the DBL arc are independent processes. The essential point of this model is that the arc moves in the plane of the spherical outer surface of the gastrula. It follows, first, that the curvature of the outer DBL arc surface is that of the surface over which the arc moves. Second, this means that the inward movement of cells entering the DBL, "bending over the arc and contributing to the formation of the archenteron roof, does not shape the DBL in sagittal section, except for the archenteron roof lengthening, which is based on the radial and planar intercalation of the archenteron roof cells (Keller, Tibbets, 1989; Keller, 1996).

The model based on these assumptions is given in Fig. 4C, which represents a sagittal section of the DBL arc at a stage at which the DBL forms a topological fold, at which point the archenteron roof becomes distinguishable from the archenteron bottom. The direction of cell flows, shown in the figure by the arrow, is in the outer surface plane of gastrula. This is the only orientation of a mass cell movement, whatever might be its driving
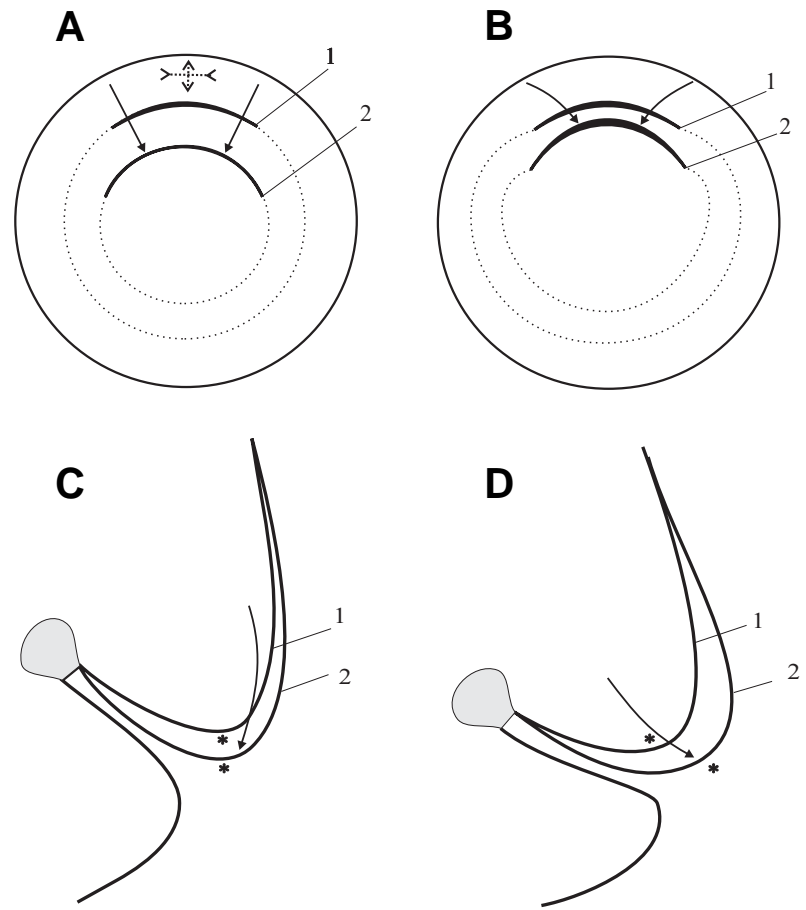

Fig. 4. Scheme illustrating the difference between dorsal blastopore lip (DBL) movement and movement-shaping modes. (A,C) Movement mode; (B,D) Movement-shaping mode in planar $(A, B)$ and sagittal $(C, D)$ views. $(1,2)$, The successive $D B L$ contours, shown by solid arrows are the directions of mass cell flows; asterisks, bending points between the outer and inner DBL surfaces; shading, the archenteron botttom; dotted line, $M Z$ circumference; dotted arrows in $(A)$, the intercalation movement vectors. For other explanations, see text. 
forces, that provides archenteron roof lengthening without DBL shaping. One of the predictions of this model is that the archenteron roof lengthening is an even process having the linear dependence on DBL movement in the embryonic surface plane.

Fig. 3D shows the dependence of the linear archenteron roof length (gi) on the DBL linear length (larc). The connection between these characters is not even, but rather a threshold relationship, just as that between the arc linear height and length, shown in Fig. 3B. It follows that in the sagittal plane the DBL has the same shaping as in the plane of the outer embryonic surface. Note the contrast of $M Z$ lengthening in the course of epiboly, shown in Fig. 3A, as compared to the jump in the archenteron roof length at a threshold value of the DBL length on the embryonic surface.

The movement-shaping model of DBL development in the sagittal section corresponding to this type of dynamics is shown in Fig. 4D. It differs from the gastrulation model, shown in Fig. 4C, in the same respect in which the model, shown in Fig. 4B for the planar movement-shaping, differs from that shown in Fig. 4A. The distinction is that the direction of mass cell flows (the arrow in Fig. $4 \mathrm{C}$ ) is permanently adjusted to the shape of the DBL contour molded by the mass cell movement. Therefore, the predominant direction of the mass cell movement is not in the outer surface plane corresponding to that of epiboly. Rather, it deviates towards the bending point between the outer and inner DBL surfaces, as shown in Fig. 4D. On the other hand, as the cells enter the bending zone between the outer and inner DBL surfaces, their inward movement changes the shape of the bending zone surface so as to increase its local curvature. This leads to a situation, shown in Fig. 4D, in which the archenteron lengthening weakly correlates with the DBL bending point movement in the embryonic outer surface plane. Obviously this corresponds well to the previously mentioned increase in the DBL arc height which is independent of that in the arc length (see Fig. $3 \mathrm{C}$ ). It follows that the archenteron roof lengthens primarily on the expense of DBL bulging in which the bending point moves not in the outer surface plane of the whole gastrula, but rather in a direction that is almost perpendicular to the plane of the epiboly movement.

In fact, this is not the movement of a point, that is, not the movement of the implicit dynamical system, but movementshaping in which the change in the position of a given point arises as a result of shaping the bending zone between the outer and inner DBL surfaces which acquires and increases its curvature on its own. The bulging of the DBL that is missed by the marking experiments (Keller, Tibbets, 1989) and SEM observations (Hardin,

TABLE 1

\section{SPECIFIC GROWTH RATES AND THE COEFFICIENTS OF VARIATION $\left(C_{v}\right)$}

\begin{tabular}{lcc} 
& $\boldsymbol{C} \boldsymbol{v}$ & $\begin{array}{c}\text { Specific growth } \\
\text { rates 2-3 }\end{array}$ \\
\hline aarc & $35 \%$ & 1.3 \\
harc & $54 \%$ & 2.5 \\
larc & $28 \%$ & 0.85 \\
giav & $61 \%$ & 3.26 \\
gil & $43 \%$ & 1.72 \\
ma & $13 \%$ & 0.32 \\
baw & $15 \%$ & -0.1 \\
lyp & $11 \%$ & -0.12 \\
\hline Cv, coefficient of variation at fixation 2 \\
(crescent-like stage)
\end{tabular}

Keller, 1988; for review see Beetschen, 2003) has a critical importance to the amphibian gastrulation, as it allows for the selfenhancing accumulation and commitment of cells of the future notochord (Cherdantsev, 2003). At later stages, after the segregation of the notochord anlage has completed, the connection between the movement of the DBL cells and shaping of the DBL arc is lost (Cherdantsev, Scobeyeva, 1996). Therefore, the course of the normal gastrulation seems to regress to that of the epiboly, shown in Figs. 4A and $\mathrm{C}$, in which the driving forces of the archenteron roof lengthening are the radial and planar intercalation of the archenteron roof cells.

\section{Morphological variation as a measure of rates of direc- tional morphological changes}

The origin of a new domain, the DBL, leads to a new time scale of morphological changes that is manifested in variation. The coefficients of variation ( $C V$ ) of metric characters in the same egg clutch of embryos, measured at consecutive gastrulation stages, are given in Fig. 5A: the vegetal side views of embryos are shown under the abscissa. To estimate the variability scales, we have to recall that in the adult organisms the "intangible" variation is that "which cannot be attributed to external circumstances, but is attributed, in ignorance of its exact nature, to 'accidents ' or 'errors" of development as a general cause" (Falconer, 1981), shows up mostly in the within-individual variance of repetitive morphological characters whose Cvs are of the order of $15-20 \%$ (see, for example, Reeve, Robertson, 1954). If the Cvvalues exceed $20 \%$, both the zoologist and geneticist would be inclined to suspect sampling error. However, in our case the sample is homogenous enough both in genetic origin, as the embryos under consideration developed from the eggs of the same clutch and in environmental conditions of their development. This can be verified by the fact that $C$ vof the egg diameter, whose mean value is not subject to prominent directional change in embryogenesis, depends primarily on the age of females and, consequently, being a property of the adult organism, is only $5 \%$ - the usual order of magnitude for the variation of adult characters that are moderately important as determinants of natural fitness in a genetically homogeneous sample (Falconer, 1981).

The metric characters, as follows from"Fig. $5 \mathrm{~A}$, can be subdivided into two groups. One, shown by gray in the figure, refers to those parts of the gastrula that have been formed at the onset of gastrulation and continue the epiboly movement. The other group, shown in black, includes the characters concerning with DBL domain morphogenesis. The characters of the first group have a Cvat a level of $10-20 \%$, while in the second group the Cvvalues exceed $20 \%$.

Matching the difference in the amount of variance to that in the rates of their changes in time makes it clear that the $C$ vdifferences correspond to the rates of morphological changes. Table 1 illustrates this. The specific rates of morphological changes were calculated, for each morphological variable $X i$, as $(X i n+1-X i n) /$ $X$ in, where $X i n+1$ and $X$ inwere the mean values of $X$ i, corresponding to the preceding $(n)$ and subsequent $(n+1)$ fixations of the embryos of the same egg clutch: $n$ corresponds to the second fixation, shown in Fig. 5A (crescent-like DBL), while $(n+1)$ corresponds to the third fixation, shown in Fig. 5A (semicircular $\mathrm{DBL}$ ). The $C v$ values shown in the table refer to the second 
fixation, that is, to the onset of DBL shaping. The correlation between the $C v$ values of morphological variables and specific rates of morphological changes that occur between the crescentlike and semicircular DBL is very high and statistically significant $(r=0.99, p<0.001)$.

Since Darwin, variability increase has been known as a criterion for evolutionary novelty (Cherdantsev et al., 1996). In ontogeny this would have been extraneous and the only explanation may be that morphogenesis shares essential features of selforganization. The origin of a new scale of variation in the DBL domain, whatever might be its developmental history, is possible only if new morphogenetic interactions appear. The point is that morphogenesis behaves in a way as if the appearance of this domain would have been really new and uncalculated event comparable to the appearance of a new niche in the ecological system. At the later stage of the DBL development (semicircular blastopore, see Fig. 5A) the variability decreases, as if the developing system "has adapted" to the new domain.

In fact, the formation of the semicircular blastopore means, as the DBL acquires a spatially homogeneous shape, that the shaping of the DBL is completed. On the other hand, as it follows from the independent experimental data, this is a stage at which in Ranaembryos the DBL stops recruiting new cells into the dorsal archenteron roof (Cherdantsev, 2003). Thus, the variation in metric characters of the DBL domain remains at a high level until the mass movement of the DBL cells is concerned with shaping of the DBL arc and recruitment of the new cells into this shaping process. The movement of a given area is the movement-shaping if and only if, the new cells are recruited into its movement.

It is the recruitment of new cells that allows for the increase in the planar and sagittal curvature of the DBL arc additional to that would have followed from its latitudinal position in the whole gastrula (see Figs. 4B, D). It follows that that the additional between-individual variation in the arc characters is due to withinindividual differences being a direct consequence of the spatial heterogeneity of the arc shape in the onset of gastrulation.

The main question is, why does the heterogeneity in the spatial shape increases the rates of morphological changes? The only possible explanation is that the DBL movement is subject to the movement-shaping equivalence principle that the spatial series of differently shaped areas correspond to the time succession of the same area shaping (Cherdantsev, 2003). The new morphogenetic interactions that characterize the morphological structures in status nascendiarise as a result of positive feedback between shaping the embryonic area at random and accelerating the movement of this area in the same direction. In microscopic systems the positive connection between the random fluctuations of microstates and the rates of directional changes at the macroscopic level is a criterion that the dynamical system is far from thermodynamic equilibrium and close to the self-organization threshold (Prigogine, 1980). However, insofar as morphogenetic systems are not microscopic, but rather macroscopic ones at all organization levels (see Gordon, 1999), the positive connection between the variation and rates is possible only provided that the relationship between the different areas of a structure is as between the successive macroscopic states of the same area. Then, the spatial heterogeneity on the macroscopic scale becomes a criterion of a morphogenetic system being far from equilibrium.
Thus, the connection between the scale of morphological variation and rates of morphological changes is a direct consequence of the positive feedback between the movement of a given area and recruitment of cells from surrounding areas into this movement. The mathematical mechanism connecting the amount of variance and rates of directional changes in metric characters concerned with form is as follows. The reasoning is similar to that stated in the Introduction, except that under consideration is not an imaginary example, but the real one shown in Fig. 5B. Shown in this figure are the previous (grey) and actual (black) shapes of the DBL arc in the vegetal view of a gastrula. The actual arc height, harc, can be partitioned into components harc ${ }^{m}$ and harc corresponding to the shaping components of DBL arc movement in its medial ( harc $^{\text {m) }}$ ) and lateral ( harc) regions. These are the shaping components of the same movement leading to the increase in the arc curvature, each having its own variance, because, as shown in Fig. 5B (cf. the positions of the previous and actual arc contours), the curvatures of medial and lateral arc components increase in different ways. Note that this shaping model presumes that the change in the harc value is independent of that of other characters (aarc and larc) that are concerned with arc planar shaping.
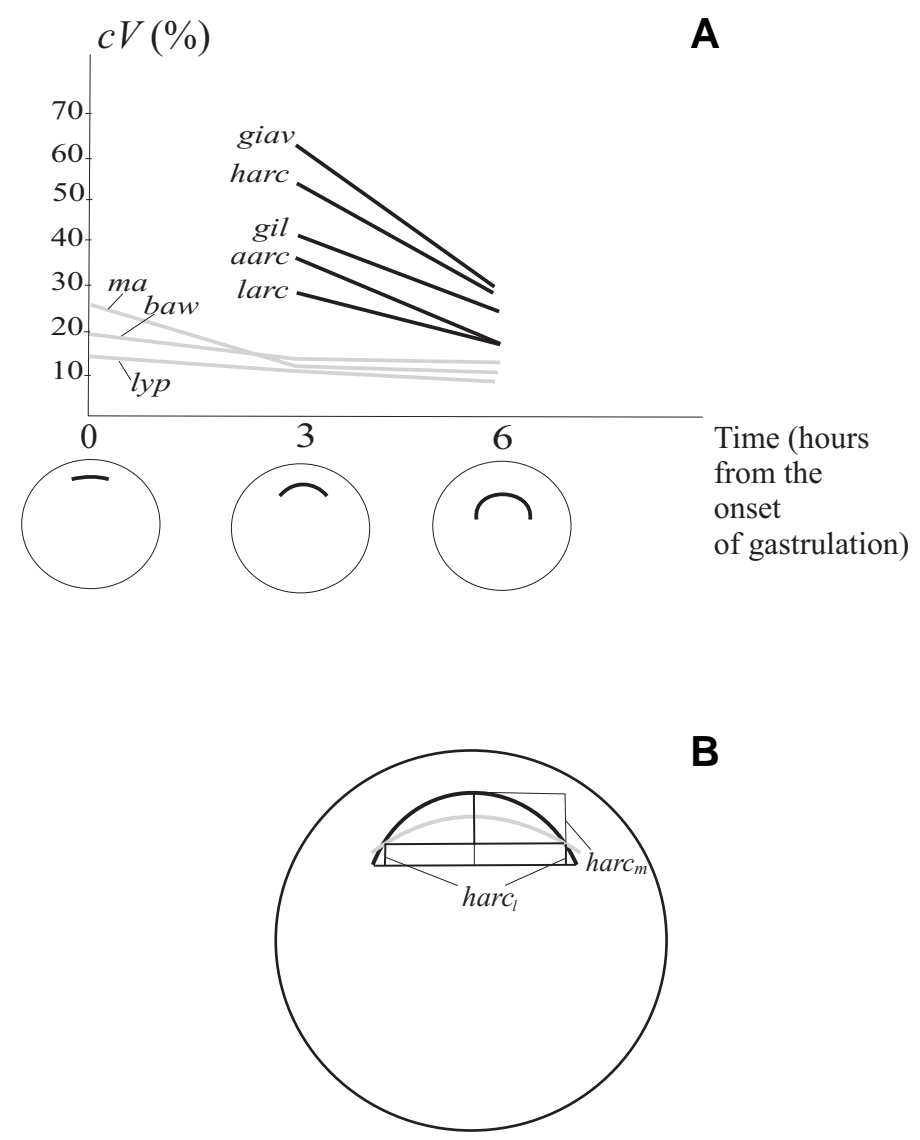

Fig. 5. Variation dynamics and spatiotemporal structure of variables concerned with shaping. (A) Coefficient of variation (cV) of metric characters (ordinate) as dependent on time (abscissa). Shown under abscissa are dorsal blastopore lip (DBL) contours. (B) Scheme illustrating the partitioning of metric characters into spatial components with harc and harc ${ }_{m}$ corresponding to the previous (grey) and subsequent (black) $D B L$ shaping stages. 
Whether or not the partitioning of a character influences the amount of its variance depends on whether there is a correlation between its components (Wright, 1923). The only assumption by which the partitioning components may correlate with one another is that the lateral and medial arc components correspond to the succession of changes in the whole arc shape, as shown in Fig. 5B: the shape of lateral components is closer to that of the whole DBL at the onset of its formation, while the medial component corresponds to the next step of DBL shaping. The point is that, as these components correspond to the consecutive steps of the same mass cell movement leading to the increase in the DBL arc curvature, any occasional increase in the contribution of medial component in the whole arc shape is at the expense of adjusting the neighboring (lateral) component to the median component curvature. In other words, any shift in the medial-to-lateral proportion of the arc regions presumes the increase in the curvature of the whole DBL arc. Then, $\sigma^{2}$ harc $=\sigma^{2}$ harc $^{m}+\sigma^{2}$ harc $+\operatorname{Cov}\left(\right.$ harc , harc $\left.^{\prime}\right)$ + Cov ( hard, harc'), where both covariance components have positive values.

To make this point clearer, imagine that the movement of the arc over the outer embryonic surface has no shaping component. Then, as shown in Fig. 4A (in contrast to Fig. 4B), each point of the arc would move centripetally along the radii of the arc curvature. If it were the case, the covariance of medial and lateral arc regions would have been equal to zero.

Thus, the positive connection between the scales of morphological variation and rates of morphological changes arises as a result of the fact that characters of embryonic areas subject to the movement-shaping equivalence have the same basic structure of their own. The components of this structure are the shaping components of a moving area whose different parts are successively recruited into the same morphogenetic movement.

Indeed, the structured characters are not the characters in the strict Cartesian sense and the variation dynamics depends neither on their choice, nor on their number. In particular, the positive movement-shaping feedbacks allowing for the increase of developmental variation and acceleration of developmental rates should not be confounded, in spite of mathematical similarity, with the effect of the positive correlation of characters whose changes jointly contribute into the same morphological change. In the frog gastrulation the increase of variability is at the stage (the crescent-like blastopore, see Fig. 5A) when the coefficients of correlation between the characters concerning with the DBL morphology have moderate values (Cherdantsev, Scobeyeva, 1996; Cherdantsev, 2003). At the earlier stage of gastrulation, when the characters concerning with shaping of the DBL arc vary almost independently from one another, the amount of variance is even higher then at the crescent-like blastopore stage (data not shown).

This favors the point that the amount of dynamical variables and complexity of their relationships are not of a critical importance. The positive connection between the variation and rates seems to be inherent to every single character structured in the sense outlined above. In any case, this is an argument favoring the origin of morphogenetic mechanisms from the self-organi- zation processes on a scale of the mass cell movements subject to the movement-shaping equivalence (for the details see Cherdantsev, 2003).

\section{Acknowledgements \\ This study was supported by RFBR project N05-04-48847A.}

\section{References}

BEETCHEN J. C. (2001). Amphibian gastrulation: history and evolution of a 125 year-old concept. Int. J. Dev. Biol. 45: 771-795.

CHERDANTSEV V. G. (2003). Morphogenesis and evolution. M.: KMK Ltd Press, (In Russian).

CHERDANTSEV V. G. and SCOBEYEVA V. A. (1994) The morphological basis of self- organization. Rivista Biology Forum. 87: 57 - 85.

CHERDANTSEV V. G., KRESLAVSKY A. G., SEVERTSOV A. S. (1996) Episelective evolution. Evolutionary Theory (van Valen ed.). 11: $69-87$.

CHERDANTSEV V. G., SCOBEYEVA V. A. (1996) On Gurwitsch's Morphogenetic Law. In Biophotonics (L. V. Beloussov, F. A. Popp. eds.). Moscow, Bioinformservices, $53-70$.

FALCONER D. S. (1981) Introduction to Quantitative Genetics (Second Edition). Longman, London and New York.

FRAGA M. F., BALLESTAR E., PAZ M. F., ROPERO S., SETIEN F., BALLESTAR M. L., HEINE-SUNER D., CIGUDOSA J. C., URIOSTE M., BENITEZ J., BOIXCHORNET M., SANCHEZ-AGUILERA A., LING C., CARLSSON E., POULSEN P., VAAG A., STEPHAN Z., SPECTOR T. D., WU Y. Z., PLASS C. and ESTELLER M. (2005) Epigenetic differences arise during the lifetime of monozygotic twins. Proc Nat/ Acad Sci USA. 26;102(30):10604-9.

GORDON R. (1999) The Hierarchical Genome and Differentiation Waves: Novel Unification of Development, Genetics and Evolution. Singapore: World Scientific and London: Imperial College Press, $1836 \mathrm{p}$.

GURWITCSH A. G. (1914) Der Vererbungsmechanismus der Form. Arch. Enwicklungsmech. des Organismen. 39: 516 - 577.

HARDIN J., KELLER R. E. (1988) The behavior and function of bottle cells during gastrulation of Xenopus laevis. Development. 105: 211-230.

JAEGER J., SURKOVA S., BLAGOV M., JANSSENS H., COSHMAN D., KOZLOV K. N., MANU, MYASNIKOVA E., VANARIO-ALONSO C. E., SAMSONOVA M.,. HARP D. H and REINITSZ J. (2004) Dynamic control of positional information in the early Drosophila embryo. Nature. 430: 368-371.

KELLER R. E., TIBBETS P. (1989). Mediolateral cell intercalation is a property of the dorsal, axial mesoderm of Xenopus laivis. Dev. Biol. 131: 539-549.

KELLER R. E. (1996) Holtfreter revised: unsolved problems in amphibian morphogenesis. Dev. Dynam. 205: 257-264.

MARTIN G. M (2005) Epigenetic drift in aging identical twins. Proc Nat/ Acad Sci USA 26;102(30):10413-4.

PRIGOGINE I. (1980) From being to becoming. Time and complexity in the Physical Sciences. W. H. Freeman and Co, N. Y.

REEVE E. C. R., ROBERTSON F. W. (1954). Studies in quantitative inheritance. VI. Sternite chaeta number in Drosophila: a metameric quantitative character. Z. induct. Abstam. -u. Vereblehre. 86: 269-288.

SCOBEYEVA V.A., CHERDANTSEV V.G. (1999) Dorsoventral polarization of gastrulation movements in common spadefoot toad (Pelobates fuscus L.) embryos: reconstruction of the mechanism based on the analysis of individual variability. Ontogenez (Russ. Journ. Devel. Biol.). 30: 436-447.

SULLIVAN, M.S., ROBERTSON, P.A. and AEBISCHER, N.A. (1993). Fluctuating asymmetry measurement. Nature 361:409-410.

WADDINGTON C. H. (1940) Organizers and Genes. Cambridge University Press.

WOLPERT L. (1969) Positional information and spatial pattern of cellular differentiation. J. Theor. Biol. 25: $76-89$.

WRIGHT S. (1923) The theory of path coefficients. Genetics 8:239-255. 